ISBN: 978-980-427-021-5

\title{
Influencia de la Calidad de Vida en el rendimiento del estudiante universitario
}

\author{
Sonia Durán¹, Ronald Prieto², Jesús García ${ }^{3}$
}

\section{Resumen}

La calidad de vida es la percepción subjetiva del individuo frente a las oportunidades que le brinda el entorno donde se desarrolla para satisfacer sus necesidades personales y laborales. Por ello la presente investigación está orientada a analizar la influencia de la calidad de vida en el Rendimiento del estudiante universitario en el contexto colombiano. Se fundamentó en un enfoque positivista, con un diseño de investigación no experimental, de campo, transversal; y un tipo de estudio descriptivo. La población estuvo constituida por los estudiantes que hacen vida en la Corporación Universitaria Latinoamericana, siendo los informantes clave cuarenta (40) jóvenes cursantes del séptimo semestre de Ingeniería de Sistema de la misma institución. Los resultados manifestaron que la calidad de vida interviene de manera determinante en el rendimiento estudiantil, bajo las perspectivas psicológicas, biológicas y socioeconómicas, y los factores individuales que tienen mayor influencia son: la disposición, las habilidades, actitudes y capacidades para el rendimiento, estos factores no pueden desvincularse del medio ambiente social al cual pertenece el individuo, por ello se asume que

1 Doctora en Ciencias Gerenciales. URBE (2010). MSc. en Gerencia de Recursos Humanos URBE 2001. Socióloga, LUZ, 1991. Investigadora en ciencias sociales, talento humano, gestión organizacional, asesora de procesos organizacionales, docente investigadora de la Universidad de la Costa, CUC, Barranquilla-Colombia. Correo electrónico: Soniaduran547@hotmail.com.

2 Posdoctor en Gerencia de las Organizaciones (URBE-Venezuela). Doctor en Ciencias Gerenciales (URBE- Venezuela). Magíster en Gerencia Empresarial (URBE, Venezuela). Economista (LUZ, Venezuela). Profesor-Investigador, editor de la Revista Desarrollo Gerencial y Decano de la Facultad de Administración y Negocios de la Universidad Simón Bolívar. Barranquilla-Colombia Investigador adscrito al Grupo: Innovación y Desarrollo Empresarial (GIDE) Correo electrónico: rprieto1@unisimonbolivar.edu.co; ronaldprieto1@hotmail.com

3 Posdoctor en Gerencia Pública y Gobierno y en Gerencia de las Organizaciones (URBE, Venezuela). Doctor en Ciencias Gerenciales (URBE, Venezuela). Magister en Gerencia de Mercadeo (URBE, Venezuela). Ingeniero Industrial (LUZ, Venezuela). Profesor-Investigador de la Universidad Simón Bolívar. Barranquilla-Colombia. Investigador adscrito al Grupo: Gestión Organizacional (GO). Correo electrónico: jesusgarcia99@gmail.com. 
el rendimiento es condicionado y de diversas dimensiones, tales como la familia, el medio ambiente, la socialización y la auto eficacia. Se concluye que los factores de mayor relevancia en la calidad de vida del estudiante son: tener una vivienda cómoda, alimentarse diariamente y en forma balanceada, gozar de buena salud, tener posibilidad de recreación, contar con instalaciones cómodas y agradables con un ambiente integrador al proyecto educativo.

Palabras clave: calidad de vida, medio ambiente, rendimiento estudiantil.

\section{INTRODUCCIÓN}

En la actualidad la búsqueda de niveles deseables y sostenibles de mejoramiento de la calidad de vida, como un aspecto de la cultura organizacional, es una inquietud que, con intereses y puntos de vista diversos, han manifestado los estudiantes, en virtud de las incertidumbres y problemas socioeconómicos que enfrenta el País. En tal sentido se ha involucrado el interés y esfuerzo por medirla desde una perspectiva integral y holística, conectándola con la búsqueda de excelencia en los aspectos esenciales de la existencia humana, de manera particular en el campo del rendimiento, la salud y el bienestar social, el cual adquiere su mayor operatividad.

Hernández y Prieto (2016) expresan que la cultura organizacional es un tema que sigue dando respuestas a muchos de los problemas que poseen las organizaciones a nivel internacional, nacional, regional y local. Los gerentes se cuestionan sobre el impacto que tiene la cultura en diversos procesos organizacionales, tales como, la innovación, la toma de decisiones, el manejo de conflictos y el trabajo en equipos, y la calidad de vida, entre otros. Cuando se llega a conocer la naturaleza cultural de las organizaciones, se comprende cómo sus miembros interpretan la realidad y reaccionan ante ella.

Asimismo, para Prieto, Emonet, García y González (2015) la estrategia para el cambio en la organización deberá estar enmarcada en una cultura organizacional sólida, que permee todo el contexto institucional, haciendo relevante que se asuman situaciones cambiantes, requiriendo procesos de comunicación, educación, participación y apoyo en el personal, que propicien mejores condiciones de vida.

En este orden de ideas, Diener (2006), considera que la calidad de vida de una persona tiene su origen en el bienestar subjetivo, el cual se alcanza por medio del establecimiento, acercamiento y cumplimiento de las metas, en este sentido la acción de la persona en lograr el bienestar, depende de sus propias capacidades, de las posibilidades de alcanzar las metas y 
de la valoración que tenga de la sociedad.

Dentro de este marco, la calidad de vida se concibe como un concepto relativo, el cual depende de la percepción del individuo y de cada grupo social y de lo que supongan como la situación ideal de bienestar, tomando en cuenta su acceso a un conjunto de bienes y servicios, así como al ejercicio de sus derechos y al respeto de sus valores; es así como cada grupo social identifica las tendencias en materia de bienestar.

Aunado a ello Nussbaum y Sen (2004), establecen que la búsqueda de la calidad de vida sea en pro de lograr un desarrollo humano capaz de facilitar en el individuo la expansión de sus opciones no sólo materiales, sino aquellas que despliegan sus capacidades intelectuales, artísticas, espirituales, de participación en una comunidad y en un sistema político que lo reconoce y le brinda posibilidades de amar, ser y tener. Sostiene que Libertad y Desarrollo, condiciones y oportunidades son dimensiones esenciales de la calidad de vida.

Cabe destacar que una situación relevante del proceso individual y social desarrollado en el ambiente universitario es el rendimiento estudiantil, el cual se presenta como uno de los problemas más dinámicos para el momento que vive el país; razón por la cual, se ha pensado que algunos elementos involucrados con la calidad de vida tienen alguna relación con el rendimiento, entre ellos se distinguen; poca preparación académica, falta de confianza en sí mismo, hábitos negativos, no tener claro su proyecto de vida, carencias afectivas, desmotivación, entre otros.

Igualmente, se han considerado los antecedentes en la familia de origen de estos estudiantes, que podrían ser el reflejo de las manifestaciones observadas. Tales son las conductas de quienes han manifestado, ausencias de cariño y realimentación emocional positiva, presiones económicas; entre otras, aunado al hecho de no haber buscado ayuda o soporte emocional anteriormente, que desde la universidad se han creado programas y procesos grupales y particulares, en virtud de dar respuesta en forma rápida y efectiva a estas situaciones planteadas, y que afecta de forma muy particular a los estudiantes.

Bajo este contexto, se percibe la calidad de vida del estudiante como eje fundamental para lograr un alto rendimiento, definido el rendimiento estudiantil como el progreso alcanzado por el alumno en función de los objetivos programados; por otra parte, es la calidad de la actuación de un alumno con respecto a un conjunto de habilidades y destrezas provocadas por un proceso de aprendizaje sistemático y expresado en una calificación. 
En este sentido, Palacios (1990), citada por Duran y Parra (2016), indican que los estudiantes universitarios constituyen una población de relevancia e interés para la realización de estudios de bienestar y salud, puesto que ellos son un grupo poblacional accesible y homogéneo que se puede identificar y acceder con facilidad y ocupan una posición significativa en la vida pública y en determinados casos en muchos aspectos de la vida social y universitaria.

Por otro lado, Sen (2004) sostiene que la calidad de vida está en los funcionamientos (habilidad de una persona para hacer actos valiosos) y la capacidad (combinaciones alternativas que una persona puede lograr). Esto hace que la medición de necesidades básicas, vaya quedando un poco en segundo plano; puesto que considera el autor que se trata de evaluación, valoración de funcionamientos y capacidades que tiene que ver con elementos constitutivos de la persona y no con bienes o recursos primarios.

De acuerdo a los aspectos evaluativos, ponderaciones, elecciones y valoraciones en términos de funcionamientos y capacidades; el enfoque de Sen (2004) permite ubicarlo en una perspectiva cualitativa y no cuantitativa, subjetiva y no objetivista, en cuanto a caracterizar su enfoque sobre el bienestar. Se distinguen funcionamientos elementales (estar nutrido, tener buena salud) o más complejos (alcanzar la auto dignidad o integrarse socialmente). Valoración de ventajas individuales y sociales van a tomar en cuenta la ponderación que los distintos individuos le dan a los funcionamientos.

En este ámbito se han desarrollado estudios sobre los factores asociados a la efectividad del estudiante, haciendo énfasis en aquellos que tienen mayor efecto, involucrándose nuevas áreas con un carácter menos académico y más vinculado con la formación integral de la persona y la mejora de su calidad de vida, éstas pasan a formar parte de un proceso de apoyo y servicio del sistema educativo, y de esta forma hacer realidad la inclusión en el medio educativo de alumnos con discapacidad.

Bajo este contexto, el rendimiento estudiantil es el resultado de un proceso independiente de trabajo realizado por un individuo o grupo, es el resultado de numerosos factores procedentes de todas las capas y ámbitos de la persona, bien sean endógenos o exógenos o ambos. Aunado a ello existen diversos factores que se involucran con el rendimiento: los individuales, son factores psicológicos y biológicos, los institucionales, referidos al sistema educativo y su funcionamiento; y los socioeconómicos que provienen de la estructura social del estudiante y de los procesos que en ella se genera, en virtud de su relación con la educación. 
Cabe destacar, que desde las universidades se ha adoptado un enfoque de mejora de la calidad de vida del estudiante, ofreciendo servicios y beneficios acorde a sus necesidades, con el fin de favorecer un aumento en el rendimiento, y donde la satisfacción del usuario, se ha convertido en un criterio de máxima relevancia. A este respecto Paz, Núñez, García y Salom (2016) señalan que en las universidades se debe reconocer la capacidad de asumir el servicio a los demás, destacando la relevancia que las organizaciones académicas deben tener en la mejora de las condiciones de vida de las personas, así como, en el bienestar y progreso de la sociedad, haciéndolas más humanas, priorizando en la calidad de vida de sus integrantes.

En consecuencia, la calidad de vida se relaciona con el carácter positivo o negativo del ambiente donde se desenvuelve el individuo y sus condiciones de vida, perfilándose bajo una concepción holística donde se analiza al individuo como un ser biopsicosocial, considerando el contexto en el cual viven las personas, vinculándolas con sus necesidades, sus expectativas y los elementos satisfactores. Una de las condiciones de la calidad de vida inherente al desarrollo del individuo es el medio ambiente y la falta de motivación, estos influyen ampliamente en el rendimiento estudiantil, puesto que muchos jóvenes de hogares de bajos recursos se ven obligados a trabajar en la edad en que debieran tener la posibilidad de dedicarse plenamente a estudiar, lo que estimula el abandono de los estudios o dificulta el proceso de aprendizaje.

El bajo rendimiento estudiantil se ha presentado como uno de los problemas más graves que sufre la educación universitaria, acarreando grandes consecuencias, tales como disminución del nivel académico, frustración en el estudiante y su familia y bajo bienestar personal y social.

En este orden de ideas, se presenta la necesidad de conocer la influencia de la Calidad de Vida en el de Rendimiento del estudiante universitario. Con la necesidad de proveerlo de habilidades y competencias que incidan en el incremento de la productividad de la sociedad en la que se desenvuelve, de esta manera impulsar el emprendimiento social y laboral y que estos puedan ser los empresarios del mañana. Dado que los emprendedores buscan crear un mundo donde los individuos tengan la libertad de decisión económica, de tener su propia empresa, de competir en el mercado, de tomar riesgos económicos, de obtener utilidades y disfrutar los resultados de sus decisiones económicas. 


\section{FUNDAMENTO TEÓRICO}

\section{La calidad de vida como fundamento del bienestar social: indicadores generales del desarrollo del individuo}

El interés por la calidad de vida existe desde tiempos remotos, aunque no existían estudios científicos sobre la variable, se ha sugerido que la aparición de la "calidad de vida" como un indicador del desarrollo del individuo se dio en la década de los sesenta junto con los movimientos sociales y las luchas contra la desigualdad, el deterioro de las condiciones de la vida urbana y del medio ambiente en los Estados Unidos y Europa. Por otra parte, hubo algunas intervenciones del gobierno en la denominada "lucha contra la pobreza", al respecto, Rivas (2001) señala que la primera personalidad pública que utilizó el término fue el presidente Lyndon B. Johnson en 1964.

En términos generales la idea de calidad de vida comienza a popularizarse en la década de los 60 hasta convertirse hoy en un concepto utilizado en ámbitos muy variados, tales como son la salud, la salud mental, la educación, la economía, la política y el mundo de los servicios en general. Como parte de su impulso inicial los esfuerzos se orientaron a operativizar la calidad de vida, desglosándola en diferentes elementos o indicadores de tal manera de poder relacionar en un proceso sistémico, una interdependencia entre dichos indicadores. Bajo este criterio, comienzan a surgir una serie de indicadores estadísticos para medir el bienestar social de una población o servir de referencia sobre el comportamiento de condiciones objetivas, formulando un patrón de comportamiento que permitiera realizar una comparación congruente.

Pero estos patrones de medida se centraron fundamentalmente en las categorías económicas tradicionales y en la identificación de necesidades materiales básicas. Ha considerado Arostegui, (1998) que estos indicadores tuvieron su propia evolución siendo en un primer momento referencia de las condiciones objetivas, de tipo económico y social, y en un segundo momento contemplar elementos subjetivos.

Posteriormente, se generó el desarrollo y perfeccionamiento de los indicadores sociales, lo cual ocurrió a mediados de los 70 y comienzos de los 80 , originando el proceso de diferenciación entre tales indicadores y la calidad de vida, bajo ese contexto la expresión comienza a definirse como concepto integrador que comprende todas las áreas de la vida (carácter multidimensional) y hace referencia tanto a condiciones objetivas como a componentes subjetivos. 
En este orden de ideas, Cobb (2000) señala que el concepto "calidad de vida" recibe un segundo impulso a finales de la década de los ochenta y en los noventa cuando comenzaron a contemplarse nuevos elementos de análisis desde el campo de la salud, la psicología, la psicología social y la rehabilitación de personas con impedimentos y deficiencias en el desarrollo. Se incorporan en esta nueva versión valorativa factores culturales, sociales y ambientales, muchos de estos cualitativos, como las relaciones interpersonales, el crecimiento personal, la participación, la libertad, la felicidad, la percepción, la satisfacción y estrés vital, entre otros.

En referencia a lo antes expuesto, Rivas (2001) refiere que la "calidad de vida", puede comprenderse desde cuatro perspectivas a saber:

1. Calidad de las condiciones de vida de una persona.

2. La satisfacción experimentada por la persona con dichas condiciones vitales.

3. La combinación de componentes objetivos y subjetivos, o sea, la calidad de las condiciones de vida de una persona junto a la satisfacción que ésta experimenta.

4. La combinación de las condiciones de vida y la satisfacción personal ponderadas por la escala de valores, aspiraciones y expectativas personales.

Según la Organización Mundial de la Salud (OMS, 2002), la calidad de vida se define como "la percepción individual de la propia posición en la vida dentro del contexto del sistema cultural y de valores en que se vive y en relación con sus objetivos, esperanzas, normas y preocupaciones"

Por su parte Rueda (2004), acota el concepto de la calidad de vida en cuatro dimensiones, la primera se refiere al bienestar general del ciudadano en cuanto su trabajo, educación, vivienda equipamientos y sanidad, la segunda está relacionado con, la calidad ambiental en la calidad de vida y que viene representada por la calidad del ambiente atmosférico, el ruido, la calidad del agua, entre otros.

La tercera dimensión es la psicosocial, y está vinculado al ámbito interactivo del sujeto: relaciones familiares, relaciones interpersonales, ocio, tiempo libre, entre otros. Por último, la cuarta dimensión hace referencia a cuestiones de cierto orden sociopolítico, tales como la participación social, la seguridad personal y jurídica. En este orden de ideas, en la conceptualización de la calidad de vida convergen variedad de enfoques, la noción es considerada como multidisciplinaria, es conceptualizado y utilizado desde distintas disciplinas como la Economía, Sociología, Medicina, Psicología, 
Filosofía, Geografía, entre otras que se ocupan de estudiar los complejos problemas económicos, sociales, ambientales, territoriales y de relaciones que caracterizan a la sociedad.

Cada disciplina ha enfatizado diferentes aspectos y se han formulado marcos de referencia desde sus diferentes perspectivas que han contribuido a su mejor comprensión. La evolución del concepto de la calidad de vida queda plasmada, en virtud de sustentar que la "calidad de vida" sugiere comprender y analizar cómo las personas interactúan con su entorno multidimensional, destacando las dimensiones cotidianas de los individuos, en sus relaciones con otros y con los espacios económicos, sociales y ecológicos.

Bajo este contexto, por medio de la calidad de vida el ser humano puede construir cosas en común, pensar, inventar, desarrollar nuevas formas de convivencias, sistemas económicos, políticos, sociales, empresariales, entre otros. Por otra parte, se dice, que el hombre es un ser productivo vinculado en el ambiente psico-social, considerando los valores como un proceso en el cual se obtiene más de lo esperado; ajustándose el comportamiento a los objetivos, propósitos y metas establecidas en su proceso psico-social, vinculado al desarrollo de habilidades y competencias en pro de optimizar su rendimiento estudiantil.

Atendiendo a estas consideraciones, Duran, Parra y Márceles (2015), relacionan este rendimiento con el proceso de educación y formación como uno de los principales factores que diferencian a los emprendedores de los no emprendedores. Consideran los autores que en el contexto educativo universitario, se perfila el emprendimiento desde un enfoque de desarrollo humano integral, el cual permite a la comunidad educativa construir conocimientos y desarrollar hábitos, actitudes y valores necesarios para generar acciones orientadas al mejoramiento personal y a la transformación del entorno y de la sociedad, al mismo tiempo, dar soluciones a las necesidades humanas presentes en la comunidad, con un sentido de ética y responsabilidad social y una perspectiva de desarrollo sostenible.

\section{DIMENSIONES DE LA CALIDAD DE VIDA: PRINCIPIOS GENERALES DEL BIENESTAR SOCIAL}

La calidad de vida, tiene diferentes concepciones, puesto que se relaciona con el carácter subjetivo de una persona o grupo de personas, para poder analizar las dimensiones de la calidad de vida es necesario conocer las posturas de algunos expertos en el tema. En este sentido, Nussbaum y Sen (2004), sostienen que, para medir y definir la calidad de vida de las 
personas, deben considerarse los aspectos sociales o humanos, tales como la expectativa de vida, la educación, la salud, la satisfacción en el trabajo, la dignidad, las relaciones laborales, familiares y los valores que permiten presuponer que la vida es más que un conjunto de relaciones comerciales.

Dentro de este marco, según la Organización Mundial de la Salud (2002), citado por Duran y Parra (2016) la calidad de vida, es la percepción que un individuo tiene de su lugar en la existencia, en el contexto de la cultura y del sistema de valores en los que vive y en relación con sus objetivos, sus expectativas, sus normas, sus inquietudes. Dicho concepto, surge cuando las necesidades primarias básicas han quedado satisfechas con un mínimo de recursos, se relaciona con algunas dimensiones, tales como la salud, vivienda, consumo de alimentos, seguridad social, ropa, tiempo libre, derechos humanos, entre otros.

Bajo este contexto la calidad de vida para Gómez y Sabeh (2003), es la combinación de las condiciones de vida y la satisfacción personal ponderadas por la escala de valores, aspiraciones y expectativas personales. Es decir, cada individuo o grupo asume su posición con respecto a la calidad de vida, relacionándolo con la satisfacción y el bienestar. Atendiendo a estas consideraciones las dimensiones de la calidad de vida se pueden clasificar en: individuales, sociales y ambientales, y para sustentar estas dimensiones se analiza la posición de algunos expertos con respecto al tema.

En este orden de ideas, Pichardo (1998) reconoce ocho dimensiones de la calidad de vida, las cuales clasifica en: a) bienestar emocional, incluye (seguridad, espiritualidad, felicidad), b) relaciones interpersonales (intimidad, afecto, entorno familiar), c) bienestar material (propiedades, posesiones, seguridad financiera), d) desarrollo personal (educación, destrezas, competencias personales), e) bienestar físico (salud, nutrición, recreación), f) autodeterminación (autonomía, control personal), g) inclusión social (aceptación, status, roles) y h) derechos (privacidad, debido proceso, elecciones).

Por otro lado, Schalock y Verdugo (2003) propusieron un modelo compuesto por dimensiones e indicadores centrales de una vida de calidad y establecen principios para entender la calidad de vida y sugieren tres niveles que afectan la calidad de vida, tales como: micro sistemas (crecimiento personal y desarrollo de oportunidades), meso sistemas (técnicas de mejoras del programa y del entorno) y macro sistemas (políticas sociales). Aunado a ello, Sen (2004) considera que la calidad de vida de una persona esta intrínsecamente relacionada con su bienestar, es decir el estado del ser de la persona, evaluando las dimensiones desde la perspectiva de su propio 
bienestar personal, lo cual finalmente se traduciría en un bien social; este enfoque permite evaluar el funcionamiento y desarrollo de una persona desde la perspectiva de su propio bienestar, aun cuando las fuentes de su bienestar sean externas.

Atendiendo a estas consideraciones, el estudio de la calidad está integrada a dos grandes dimensiones, la dimensión objetiva y la subjetiva, por ende, se concibe como un equivalente de bienestar en el ámbito social, existiendo diferentes modelos teórico-metodológicos para su análisis. En los modelos planteados prevalecen dos tendencias fundamentales: La primera centrada en variables objetivas externas al sujeto, correspondiéndose con un enfoque sociológico y económico, que ha tratado de medir la calidad de vida utilizando preferiblemente la metodología de los indicadores de corte sociodemográfico.

Estas posturas sugieren la dificultad de clasificar las dimensiones de la calidad de vida a los grupos de personas, puesto que estos varían de acuerdo a la percepción de los procesos de vida que tenga cada individuo, asimismo, se relaciona con el desarrollo de su vida diaria, en tal sentido la adecuación de las dimensiones de la calidad de vida se realizan en función de algunas características generales que se puedan extraer y analizar sobre un grupo o población específica. Partiendo de que "la calidad de vida es claramente un atributo de la persona, identificada como una actitud, un rasgo de personalidad, una respuesta situacional o un estado sentimental o un juicio racional, ella de alguna manera se relaciona o interviene en algunos en la mayoría de los aspectos de la vida de un individuo.

\section{RENDIMIENTO ACADÉMICO COMO RESULTADO DEL PROCESO DEAPRENDIZAJE: ENFOQUEDELDESARROLLO DEHABILIDADES COGNITIVAS}

En virtud de que el rendimiento académico se concibe como la capacidad de respuesta del estudiante frente a estímulos educativos, susceptible de ser interpretado según los objetivos o propósitos educativos preestablecidos. Se define según Conlon, Aranda y Aranda (1998), como el grado de logro o resultado de los objetivos establecidos en los programas oficiales de estudio o de la acción educativa sobre el sujeto, valorado y acreditado por el propio sistema educativo. Dentro de este marco, el rendimiento académico debe ser evaluado en función de ciertos indicadores, los cuales deben ser susceptibles de medición y comprobación.

Cabe destacar que en la actualidad existe una posición muy particular acerca de las facultades de las personas en relación a la instrucción y las 
capacidades humanas para el aprendizaje, especialmente en las orientaciones instrumentales educativas, aunado a ello se involucran indicadores tales como, condiciones y/o ambientes de aprendizaje apropiados, donde los estudiantes son capaces de alcanzar un alto nivel de dominio y de conocimiento.

A este respecto Gardner (1998), indica que las sociedades modernas han enfrentado múltiples dificultades al momento de resolver el problema educativo; puesto que han tenido que adaptarse a las formas tradicionales de transmisión del conocimiento, (el conductismo) y, por ende, a los criterios restringidos de evaluación y de aceptación de rendimiento por parte de los alumnos.

En tal sentido, los estilos de aprendizaje deben fortalecerse a través de la construcción del conocimiento, integrando las actitudes y habilidades personales, para lograr altos niveles de rendimiento académico, utilizando como base el constructivismo y el aprendizaje significativo, en virtud de ello el rendimiento sería la consecuencia de la acción integradora de las actitudes, habilidades y conocimientos que generan los estudiantes durante el desarrollo de su carrera profesional.

Bajo este contexto, el estudiante universitario es un ser desarrollado en lo físico, en lo psíquico, en lo social, capaz de actuar con autonomía en su grupo social y que decide sobre su propio destino, aprende de forma distinta a los demás, es decir, utiliza diferentes estrategias, aprende con diferentes velocidades e incluso con mayor o menor eficacia, aun teniendo las mismas motivaciones, el mismo nivel de instrucción, la misma edad o estén estudiando el mismo tema.

Cabe destacar que los avances realizados por la pedagogía experimental, referenciados en el Diccionario de psicología y pedagogía Lexus (1998) permiten llegar a un conocimiento bastante exacto de lo que un alumno aprende; por otra parte, si se mide la instrucción, además del aspecto intelectual de la educación, se pueden conocer otros factores de influencia en el rendimiento, tales como los factores emocionales y sociales.

Atendiendo a estas consideraciones Navarro (2003) describe el rendimiento académico como la expresión de las habilidades, actitudes y valores que son desarrollados por el alumno a través del proceso de enseñanza-aprendizaje, es decir son todas aquellas acciones dirigidas a la explicación e interpretación de lo aprendido y que se sintetizan en valores cuantitativos o cualitativos. 
Además, la medición del rendimiento a través de la instrucción, permite dilucidar acerca de la inteligencia de los alumnos, así como también de sus habilidades y destrezas, de su voluntad, motivación, sentimientos, capacidades, e incluso, de las condiciones sociales y culturales en las cuales se desenvuelven. De tal manera que pueden extraerse dos aspectos en el aprendizaje como indicador del rendimiento académico e intelectual del estudiante: el primero, los conocimientos adquiridos y el segundo; las prácticas y habilidades que le permitan ejecutar con facilidad operaciones, generalmente, de carácter intelectual.

Finalmente, el rendimiento académico no sólo es el resultado de un proceso pedagógico de enseñanza-aprendizaje, sino que es el resultado de la acción educativa donde se involucran diferentes elementos, y cada elemento con diferente grado de influencia sobre el mismo, entre los elementos más destacados están: La familia y el ambiente social.

\section{FACTORES QUE INFLUYEN EN EL RENDIMIENTO ACADÉMICO: OPORTUNIDADES Y EXPERIENCIA DE APRENDIZAJE OFRECIDAS POR EL MEDIO SOCIAL AL ESTUDIANTE}

Existen diversas opiniones acerca de las innumerables causas que pueden afectar al rendimiento estudiantil, además de los factores que afectan la calidad de la educación en Venezuela, a este respecto Garnica (2000), sugiere que la organización del sistema educativo, organización y selección del currículo, la formación docente, las estrategias y materiales didácticos y los libros de texto, son algunos de los factores que afectan el logro de altos niveles de rendimiento.

En virtud de los desajustes en el sistema educativo y en especial en el ámbito universitario se han realizado algunos estudios de los factores que inciden en el rendimiento del estudiante, entre los cuales Garnica (2000) incluye: la inteligencia individual, entorno socio-cultural, facilidades socioeconómicas, entre otros. Siguiendo el mismo orden de ideas, Padrón (2005) hace una clasificación de factores endógenos y exógenos, entre los endógenos o aptitudes se involucran lo que una persona puede hacer física e intelectualmente, como resultado de sus dotes e historia del desarrollo; aunado a ello las actitudes o características de la personalidad como la independencia, la motivación, la autonomía, el control de impulsos y la estabilidad. Además, se incluyen otros factores, tales como, la educación de los padres, los ingresos de la familia, la salud y su dieta.

Por otro lado, están los factores exógenos, los cuales hacen referencia a la calidad de la instrucción, la formación de los docentes, el acceso a los 
libros, el presupuesto de las instituciones educativas. En tal sentido, en el avance y desarrollo del conjunto jerárquico de habilidades y destrezas de las personas intervienen múltiples factores, los cuales pueden alterar o influir directamente en el rendimiento estudiantil y generar un estudiante con problemas de rendimiento, situación que podría originar la deserción y el rezago.

Dentro de este marco, el rendimiento académico, se caracteriza por ser un proceso complejo puesto que existe una interrelación multifactorial en el contexto educativo, bajo este enfoque, el rendimiento académico está estrechamente relacionado con dos grupos de factores: los socioeconómicos y los individuales, los cuales se explican a continuación.

\section{Factores Socioeconómicos}

Es importante analizar que el principio de igualdad de oportunidades significa que cualquier individuo pueda acceder a la educación como cualquier otro, con independencia de los factores o características tales como, la renta familiar, la clase social, la raza, el sexo o cualquier otra fuente irracional de diferencias. En este sentido, se considera que debe existir un criterio de igualdad o de equidad categórica; aunque los niveles de educación alcanzados ex-post por dos individuos sean perfectamente distintos como consecuencia, principalmente, del esfuerzo, la suerte, las preferencias individuales u otras diferencias legítimas, pero no quedarían justificados por condicionantes de tipo socioeconómico.

Bajo este contexto Palacios (1990), hace referencia a los factores socioeconómicos como aquellos con mayor tendencia a la relación con el rendimiento estudiantil, en virtud de la influencia que tienen en el proceso educativo en general, afectando las características psicológicas y el desarrollo y crecimiento biológico y social de las personas. Por ende, los inputs familiares se consideran como fuertes condicionantes de los resultados en la escuela, puesto que se relacionan con características socioeconómicas y sociodemográficas de las familias tales como la educación de los padres y sus ingresos, el tamaño de la familia o los recursos materiales en el hogar.

Establece, que los factores socioeconómicos comprenden el conjunto de indicadores sociales y económicos que caracterizan el desarrollo de un individuo o grupo, ofreciéndole la posibilidad de ocupar una determinada posición en la sociedad, así como el disfrute de bienes y servicios, del mismo modo facilita el acceso a las oportunidades. Dentro de este marco, los factores socioeconómicos tienen una estrecha relación con el comportamiento, el desarrollo de las capacidades y valores del individuo, entre los 
factores socioeconómicos que se vinculan al análisis están: el ingreso, la ocupación o situación laboral de los padres, las condiciones de vida y salud y el contexto familiar y social.

Para Padrón (2005), el bajo rendimiento académico se involucra con el proceso escolar, el cual produce un impacto psicológico en el niño expresado en sufrimiento y probables secuelas en su desarrollo, afectando su calidad de vida. El autor señala que el $20 \%$ de la población infantil presenta bajo rendimiento escolar, constituyéndose un problema por los altos costos económicos y sociales, señalando entre las causas principales el déficit de atención y los relacionados con la dislexia, y problemas asociados. Uno de los factores más importantes y hasta ahora poco considerados son las deficiencias nutricionales.

Por otra parte, Álvarez, García y Gil, (1999) involucra la escuela como el sistema de educación formal, es uno de los agentes de socialización más importantes después de la familia y ejerce una influencia benéfica sobre el desarrollo psicosocial, la adquisición y utilización del conocimiento y el progreso económico y social de los individuos y la sociedad tanto en los países desarrollados como en los países en desarrollo. Aunado a ello, las instituciones educativas tienen como fin que sus alumnos y alumnas adquieran conocimientos, habilidades, e instrumentos que los transformen en individuos socialmente activos.

\section{Factores Individuales}

Los factores individuales incluyen la disposición del estudiante, las habilidades, las aptitudes y capacidades, los cuales involucran el estímulo interno para lograr el rendimiento y el desarrollo de las habilidades y destrezas. En este sentido, la motivación individual es un elemento decisivo en el rendimiento académico, ya que el nivel de ejecución intelectual no es simplemente un asunto de habilidad personal; el nivel de motivación del estudiante constituye una explicación sobre los resultados en un proceso educativo. De esta manera, los puntajes en una prueba de rendimiento de estudiantes que tengan el mismo nivel de habilidad serán diferentes en función de su motivación; la habilidad de un estudiante que no está motivado óptimamente será subestimada en la prueba.

En este orden de ideas, los factores individuales están influenciados en gran magnitud por el desarrollo psicosocial del individuo, el cual en su etapa escolar distinguida como "laboriosidad versus inferioridad", aprende las destrezas y habilidades propias de su cultura y lo ayudan a prepararse para la vida adulta, por lo cual se convierte en un "trabajador", es decir 
se esfuerza para merecer reconocimiento en función de las exigencias del medio y de sus propios esfuerzos, para ello, es fundamental que durante los primeros años, haya adquirido confianza, autonomía y seguridad, lo que conlleva a desarrollar la autoestima.

Dentro de este marco, es importante destacar que en Colombia Bodensiek, (2010) realizó un estudio denominado el estudio, "Factores que influyen en el rendimiento escolar", publicado por la Secretaria de Educación de Bogotá, en el concluyo que entre los factores que influyen en el rendimiento se conciben el género, edad, frecuencia de estudio, hábitos, trayectoria académica, características familiares, ocupación, nivel educativo de los padres, vida familiar, clima afectivo y seguridad, uso del tiempo, prácticas de crianza y relaciones de la familia con la escuela, cultura, infraestructura, enseñanza, uso del espacio y el tiempo, organización, rutinas y normas, clima, formas de evaluación y sistemas de premios y castigos son elementos asociados al R.A.

En este sentido, el desarrollo evolutivo de las personas, es un proceso gradual que depende de las oportunidades y experiencias de aprendizaje que el medio le proporcione al niño, quien evoluciona en varias áreas o procesos a la vez, que no son independientes, sino complementarios. Por tal razón, es necesario reconocer y transformar los conocimientos, actitudes y prácticas que vulneran y afectan negativamente las condiciones de vida de los niños en las familias y en la sociedad, para poder lograr un individuo con habilidades y destrezas acordes al sistema de desarrollo profesional.

\section{Metodología de Estudio}

Esta investigación se desarrolló según criterios de la investigación de tipo descriptiva, no experimental, de campo la población estuvo constituida por 40 estudiantes de ingeniería de sistema cursantes del séptimo semestre en el periodo 2-2016, de la Corporación Universitaria latinoamericana Barranquilla, Colombia. Para la recolección de la información se utilizó la observación por encuesta, utilizando un cuestionario compuesto por 67 ítems y con diferentes alternativas de respuesta sustentándolo con la revisión bibliográfica, para hacer consideración de los índices académicos de los estudiantes.

Por otra parte, para determinar el nivel de Calidad de Vida del estudiante de ingeniería de sistema, se hizo necesaria la construcción de un baremo utilizando como base los valores totales de los resultados del instrumento de recolección de datos, donde el valor más alto posible de obtener fue de 335, y el valor más bajo es 67. Luego de esto, se establecieron tres 
criterios de medición, a saber: Alto, Medio y Bajo, por lo cual para cada uno se estableció un rango con los valores antes encontrados. El rango obtenido para cada nivel es de 89. En el cuadro 1, se puede apreciar el baremo.

Cuadro 1. Baremo

\begin{tabular}{|c|c|}
\hline CRITERIOS & RANGOS \\
\hline ALTO & $\geq 246 ; \leq 335$ \\
\hline MEDIO & $\geq 156 ; \leq 245$ \\
\hline BAJO & $\geq 67 ; \leq 155$ \\
\hline
\end{tabular}

Fuente: Duran, Prieto y García (2016)

\section{RESULTADOS Y DISCUSIÓN}

\section{Influencia de la Calidad de Vida en el Rendimiento del estudiante universitario}

Con respecto al cuestionario aplicado a los estudiantes que integraron la población objeto de estudio, se presenta las siguientes evidencias: Con respecto a la calidad de vida se presentan dos aspectos y 2 relacionados con el rendimiento.

\section{Calidad de Vida}

Tabla 1. Percepción de un ambiente agradable en la institución

\begin{tabular}{|c|c|c|}
\hline ALTERNATIVAS & FRECUENCIA & $\%$ \\
\hline Siempre & 26 & 61 \\
Casi Siempre & 7 & 16 \\
Con Frecuencia & 4 & 9 \\
Casi Nunca & 3 & 7 \\
Nunca & - & 7 \\
\hline TOTAL & $\mathbf{4 0}$ & $\mathbf{1 0 0 , 0}$ \\
\hline
\end{tabular}

Fuente: Duran, Prieto y García (2016)

Al indagar las condiciones del ambiente que se percibe en la institución, el $61 \%$ siempre lo percibe como agradable, el 16\% casi siempre agradable, el 9\% opino que con frecuencia era agradable, el 7, \% señaló que casi nunca aunado el $7 \%$ quienes afirmaron que nunca el ambiente era $\mathrm{n}$ la Corporación Universitaria era agradable. 
Tabla 2. Necesidades básicas cubiertas

\begin{tabular}{|c|c|c|}
\hline ALTERNATIVAS & FRECUENCIA & $\%$ \\
\hline Siempre & 12 & 30 \\
Casi Siempre & 5 & 12 \\
Con Frecuencia & 20 & 50 \\
Casi Nunca & 3 & 8 \\
Nunca & 0 & 0 \\
\hline TOTAL & 40 & 100,0 \\
\hline
\end{tabular}

Fuente: Duran, Prieto y García (2016)

Tal como se observa en la tabla y gráfica, en relación a las necesidades básicas del estudiante, un $30 \%$ sostuvo que siempre logra cubrirlas, un 12\% casi siempre, un 50 \% consideró que con frecuencia logra cubrir sus necesidades básicas, y un $8 \%$ casi nunca puede hacerlo. Lo que indica que los estudiantes en su mayoría cubren este aspecto tan importante de la calidad de vida, no obstante, es importante evaluar las condiciones del $58 \%$ que considero que con frecuencia y casi nunca.

Tabla 3. Nivel de calidad de vida

\begin{tabular}{|c|c|c|c|}
\hline CRITERIOS & RANGOS & ABSOLUTO & (\%) \\
\hline Alto & $335---246$ & 10 & 3 \\
\hline Medio & $245---156$ & 29 & 73 \\
\hline Bajo & $155---67$ & 1 & 24 \\
\hline
\end{tabular}

Fuente: Duran, Prieto y García (2016)

Se muestra en la tabla y grafico 3 los resultados asociados al nivel de calidad de vida del estudiante de Ingeniería de sistemas de la Corporación Universitaria Latinoamericana, se expresa que el $73 \%$ de los estudiantes, según la información registrada se encuentra en un nivel medio de Calidad de vida, un $24 \%$ se ubicó en nivel bajo y el $3 \%$ en alto nivel de calidad de vida, esto en virtud de los ítems incluidos en el cuestionario, tales como acceso a bienes y servicios, tipo de vivienda, espacios físicos, ubicación geográfica, entre otros.

Dentro este marco, para Somarriba y Peña, (2009) se perfila el concepto de calidad de vida, como una noción multidimensional, la cual incluye las condiciones en las que los individuos desarrollan su vida y además la valoración de esas condiciones desde la perspectiva de los propios sujetos. Como señalaron en su estudio Doyal y Gough, (1994), la calidad de vida denota la percepción individual que cada sujeto tiene respecto de su posición en el contexto cultural y sistema de valores en el que vive, en relación con sus 
logros, expectativas e intereses. Es un concepto extenso y complejo que considera la salud física, la situación psicológica, el nivel de independencia, las relaciones sociales, y las relaciones del medioambiente"

\section{Rendimiento del estudiante}

Tabla 4. Creatividad personal

\begin{tabular}{|c|c|c|}
\hline ALTERNATIVAS & FRECUENCIA & $\%$ \\
\hline Siempre & 28 & 70,0 \\
Casi Siempre & 6 & 15,0 \\
Con Frecuencia & 4 & 10,0 \\
Casi Nunca & 2 & 5,0 \\
Nunca & 0 & 0 \\
\hline TOTAL & 40 & 100,0 \\
\hline
\end{tabular}

Fuente: Duran, Prieto y García (2016)

Al ser consultados los estudiantes acerca de si se consideran creativos, se obtuvo que el $70 \%$ considera que siempre lo es. El 15\% casi siempre, el $10 \%$ con frecuencia y el $5 \%$ casi nunca, lo cual evidencian que más del 90\% del personal supone ser creativo.

Tabla 5. Promoción de competencias y habilidades en los estudiantes

\begin{tabular}{|c|c|c|}
\hline ALTERNATIVAS & FRECUENCIA & $\%$ \\
\hline Siempre & 7 & 17 \\
Casi Siempre & 6 & 15 \\
Con Frecuencia & 9 & 23 \\
Casi Nunca & 18 & 45 \\
Nunca & 0 & 0 \\
\hline TOTAL & 40 & 100 \\
\hline
\end{tabular}

Fuente: Duran, Prieto y García (2016)

En relación a Promoción de competencias en y habilidades en los estudiantes de ingeniería de sistema, el 45\% respondió que casi nunca, el 22\% con frecuencia, el 17,5\% siempre y el 15\% casi siempre. Esto indica que en la institución promover las competencias y habilidades del estudiante no se ha percibido como un elemento clave para elevar el nivel de rendimiento estudiantil.

De acuerdo con Erazo (2012) El rendimiento académico es reconocido por su capacidad clasificatoria y su vinculación a la promoción y evaluación de estudiantes, su expresión en notas y promedios académicos lo identifican con objetividad, pero además lo vincula con factores subjetivos y sociales, 
convirtiéndolo en una condición fenomenológica. Asimismo, considera el mismo autor que el Rendimiento Académico es entendido como el sistema que mide los logros y la construcción de conocimientos en los estudiantes, los cuales se crean por la intervención de didácticas educativas que son evaluadas a través de métodos cualitativos y cuantitativos en una materia.

Conclusiones

Analizar y definir las dimensiones de la calidad de vida de las personas incluye procesos sociales complejos, con variados componentes. Su conceptualización, debe tener en cuenta las opiniones de las personas y su medición requiere de un sistema de indicadores, sustentado en las necesidades y expectativas de un grupo social homogéneo, sin embargo, éste ha sido estudiado bajo diferentes enfoques y se ha llegado a la conclusión que es una concepción ambigua, que depende en gran medida del sistema de valores bajo el cual se ha desarrollado la persona o grupo social.

A este respecto, la calidad de vida es la combinación de las condiciones de vida y la satisfacción personal ponderada por la escala de valores, aspiraciones y expectativas personales. Es decir, cada individuo o grupo asume su posición con respecto a la calidad de vida, relacionándolo con la satisfacción y el bienestar. Por otra parte, se ha señalado que la calidad de vida de las personas antes de iniciar la educación formal, incide significativamente en la clase de estudiantes que ellos pueden llegar a ser, en lo cual influye en gran medida su desarrollo socio afectivo.

Entre los factores más determinantes se encuentran: contar con buena salud y nutrición, ya que los individuos física y psicológicamente saludables obtienen mejores experiencias de desarrollo psicosocial en la niñez temprana, asistencia regular a las instituciones; apoyo familiar para el aprendizaje, así mismo, el nivel educativo de los padres, el cual tiene un impacto multifacético en la habilidad de las personas para aprender. Eso se va promoviendo hasta la edad adulta y su pase a la vida universitaria.

Con respecto al ambiente social y familiar, se dice que el aprendizaje puede ocurrir en cualquier lugar, pero los logros de aprendizaje positivos deseados en educación se dan en entornos de calidad; que sean saludables, seguros, protectores y sensibles a los dos sexos y provean recursos y facilidades adecuadas, es decir que el rendimiento está condicionado por la calidad de vida.

Indudablemente el estudiante convive y se desarrolla en un ambiente bastante complejo donde intervienen múltiples factores, ellos se van generando desde el ambiente familiar, social y el institucional, los cuales van 
a formar y sustentar la personalidad del ser humano. En este sentido la educación y el proceso de aprendizaje de los niños y los jóvenes se apoya en la socialización, concebida ésta como el proceso donde el individuo adopta los elementos socioculturales de su medio ambiente y los integra a su personalidad para adaptarse a la sociedad, en éste intervienen no sólo las personas significativas para él, como padres o hermanos, sino también instituciones como la escuela, la iglesia, entre otros; estos son denominados agentes de socialización.

Bajo este enfoque, se adquieren las creencias, valores y comportamientos que una sociedad considera como deseables o apropiados, además el ser humano se hace miembro de la sociedad a través de este proceso, el cual conduce al desarrollo de prácticas de comunicación, relación e interacción, o, dicho de otro modo, al desarrollo de conciencia de sí y de los otros clara y equilibrada, es decir, identidad propia y sentido de los limites.

Por lo tanto, el proceso educativo es una experiencia social en la que el ser humano va conociéndose, enriqueciendo sus realizaciones con los demás, adquiriendo las bases de los conocimientos teóricos y prácticos, esta experiencia se inicia desde el ambiente familiar y se relaciona con el social y el educativo. Aunado a ello, la familia, el medio ambiente, la socialización y la auto eficacia tiene gran influencia en la búsqueda de objetivos lúdicos, de aprendizaje y estimulación.

Cabe destacar, que las condiciones económicas que acompañan a los estudiantes en su integración al medio, están relacionadas con los nuevos retos que deben enfrentar en el campo académico, entre ellos el desarrollo del proceso cognoscitivo, promover sus habilidades sociales, las competencias personales y sociales, entre otros.

Finalmente, se ha podido constatar en estudios realizados en la institución objeto de estudio, que los factores de mayor relevancia en la calidad de vida del estudiante son: tener una vivienda cómoda, alimentarse diariamente y en forma balanceada, gozar de buena salud, tener posibilidad de recreación, contar con instalaciones cómodas y agradables con un ambiente integrador al proyecto educativo; asimismo se ha destacado que los estudiantes desean tener un trabajo estable para acceder a ingresos propios.

Por otro lado, los factores de la calidad de vida que influyen en el rendimiento, en la mayoría son individuales, tales como habilidades y destrezas en las áreas a desarrollar, el desarrollo cognitivo, la timidez, la poca capacidad de integración y la socialización, y algunos socioeconómicos, tales como la salud, la alimentación, el acceso a los bienes y servicios. Aten- 
diendo a estas consideraciones, la calidad de vida se perfila como un eje fundamental para lograr altos niveles de rendimiento estudiantil en las instituciones de educación superior.

Es importante destacar, que al ser un concepto subjetivo las personas pueden diferir en mucho en la ponderación dada a los diferentes funcionamientos, ya que todos los individuos poseen cualidades diferentes, circunstancias externas diversas que influyen en su desarrollo. En este aspecto se conciben además los contextos sociales a los que pertenece el estudiante, el cual ofrece diversas oportunidades y ventajas, pero también ciertos obstáculos y restricciones, los cuales en la evaluación de la calidad de vida deben de tenerse en cuenta, en este sentido cada persona tendrá una percepción diferente de la vida y ordenara sus prioridades en la vida en relación a ello, por supuesto que es fundamental para el rendimiento académico del estudiante.

\section{REFERENCIAS BIBLIOGRÁFICAS}

Álvarez V.; García, E.; Gil, J. (1999). El rendimiento académico en la Universidad desde la perspectiva del alumnado, en Revista Española de Orientación y Psicopedagogía, 17 (10), pp. 23-42.

Arostegui, I. (1998). Evaluación de la calidad de vida en personas adultas con retraso mental en la comunidad autónoma del País Vasco. Universidad de Deusto.

Bodensiek, A. (2010). Estudio sobre los factores que influyen en el rendimiento escolar. Bogotá - Colombia: Secretaría de Educación Municipal.

Cobb, C. (2000). Measurement tools and the quality of life, redefining progress

Conlon, K., Aranda, L., Aranda, E. (1998). Teams: Estructure, process, cultura and politics. Prentice Hall.

Diccionario de Psicología y Pedagogía Lexus (1998). Editorial Planeta.

Diener, E. (2006). Guidelines for national indicators of subjective well-being and ill being. Applied Research in Quality of life, Springer Science, The International Society for Quality-oflife studies, (ISQOLS) Vol. 1, Issue 2: 151-157.

Duran S. y Parra M. (2014) Diversidad cultural para promover el desarrollo de habilidades sociales en Educación superior. Revista CES. Número 1-5. Educosta Barranquilla Colombia.

Durán, S., Parra, M., \& Márceles, V. (2015). Potenciación de habilidades para el desarrollo de emprendedores exitosos en el contexto universitario. Opción, 31(77).

Duran S. y Parra M. (2016) Condiciones Laborales y Calidad de vida en el trabajo: en Libro electrónico. Estrategias Innovadora: Afrontando los retos de la economía global. Universidad Autónoma de Tlaxcala. Tlaxcala, México, 2016 Primera Edición, 2016. ISBN: 978-607-8432-74-5. P 437-459.

Erazo Oscar A, (2012), El rendimiento académico, un fenómeno de múltiples relaciones y complejidades, Revista Vanguardia Psicológica Clínica Teórica y 
Práctica- ISSN 2216-0701 UNIVERSIDAD MANUELA BELTRÁN, Programa de Psicología, Bogotá D.C. Colombia

Gadner, H. (1998). Inteligencias Múltiples en Educación, Automind. Santiago de Chile.

Garnica Olmos, E. (2000). El rendimiento estudiantil: una metodología para su medición. Instituto de Investigaciones Económicas y Sociales. Universidad de Los Andes.

Gómez-Vela, M. y Sabeh, E. (2003). Calidad De Vida. Evolución Del Concepto y su Influencia en La Investigación y La Práctica. Instituto Universitario de Integración en la Comunidad, Facultad de Psicología, Universidad de Salamanca. España.

Hernández, O. \& Prieto, P. (2016). Cultura organizacional en cooperativas del Sector agrícola en el departamento del Atlántico. Libro. Dialogo de saberes desde las Ciencias Económicas, Administrativas y Contables. Corporación Universitaria CECAR. P.p. 1-15.

Navarro, R. (2003). El Rendimiento Académico: Concepto, Investigación y DesarroIlo, Reice: 1(2), 1-15

Nussbaum M. y Sen A. (2004). La Calidad de Vida. Fondo de Cultura Económica. México.

Padrón F. (2005). Factores De Riesgo Individual y Familiar relacionados con el Bajo Rendimiento Escolar. Tesis doctoral Facultad de Medicina, Universidad del Zulia.

Palacios de Echeverría C. (1990). El rendimiento Estudiantil en la Escuela de Educación de la Universidad del Zulia. Universidad Católica del Táchira.

Paz, A. Núñez, M., García, J. y Salón, J. (2016). Rol del liderazgo ético en organizaciones académicas. Revista Opción. Año 32. Especial número 12. Pp. 148-168

Pichardo, M. (1998). Evaluación del impacto social. La calidad de vida como meta última del desarrollo. PNDU (Ed.). Informe sobre desarrollo humano. Edic. Mundi prensa

Prieto, R., Emonet, P., García, J. y González, D. (2015). Cambio organizacional como estrategia de gestión en las empresas mixtas del sector petrolero. Revista de Ciencias Sociales. Volumen XXI, número 3. Pp. 386-402

Rivas M. (2001). Calidad de vida, indicadores de calidad de vida y la educación, Énfasis, centro dedicado a la investigación e intervención social y económica, Mayagüez, Puerto Rico.

Rueda, S. (2004) Habitabilidad y calidad de vida, en Cuadernos de Investigación urbanística, (42). 29-34 Pp.

Schalock, R. y Verdugo, M. (2003). Calidad de Vida. Manual para profesionales de educación, salud y servicios sociales. Alianza Editorial, Madrid, España.

Sen, A. (2004). "Capacidad y bienestar". En: La Calidad de Vida. Fondo de Cultura Económica. 588 Pág. México.

Somarriba, N. y Peña, B. (2009). La medición de la calidad de vida en Europa, el papel de la información subjetiva. Revista estudios de la Economía aplicada. Vol. 27-2. 